\title{
Key Success Factors for Merging Sport Science and Best Practice
}

The gap between sport science and field practice has been the subject of considerable debate. While there are numerous examples describing how poorly research often applies to the field, there are numerous success stories from which lessons can be learned. As an employee at Olympiatoppen (the Norwegian Olympic Sport Center) in the time period 1994-2019, I was continuously involved in discussions around the importance of research for sport performance. At the beginning of this century, most elite athletes and coaches at Olympiatoppen were not interested in sport science, based on the arguments that academic institutions addressed research questions of limited relevance to the elite sport community and the large majority of scientific studies were performed on young and/or untrained athletes/students. However, we were also challenged by research groups with convincing arguments based on results from short-term training interventions on more or less untrained populations, without having the ability to provide strong scientific evidence for our best practice approach. This dilemma created confusion among athletes and coaches. One notable example was that endurance athletes were strongly recommended to solely focus on high-intensity intervals, whereas low-intensity training was considered a waste of time. This was followed by a marked performance decline in many endurance sports after the 2002 Olympics, particularly for Norwegian crosscountry skiing. It was not until Stephen Seiler and his associates published a series of articles related to intensity and duration distribution in endurance athletes that the polarized training concept was "reborn." This concept detailed that $80 \%$ to $90 \%$ of the total training duration should be performed at low intensity, whereas $10 \%$ to $20 \%$ should be performed at higher intensity zones. At the same time, this knowledge was implemented when Norwegian crosscountry skiing clarified its training philosophy, based on a mix of science and best practice, together with researchers Espen Tønnessen and Øyvind Sandbakk. This period of reflection was followed by a long-term success for Norwegian winter endurance sport. The take-home messages from this story are that (1) caution should be used when sport scientists with limited practical experience provide "groundbreaking" training prescriptions for elite athletes and (2) the likelihood for success increases when training implementation is founded on an interaction between best practice and science.

Over the last few years, an increasing number of Norwegian elite practitioners has allowed sport scientists to play a more important role in athlete development and performance. This positive shift can mainly be attributed to the restructuring of Olympiatoppen's research and development department, which has a mandate today to (1) identify research problems relevant for elite sports, (2) ensure that applied research is performed in collaboration with leading research institutions, and (3) implement derived knowledge in the daily training process and coaching education programs. Accordingly, relevant research projects are facilitated, funded, and completed. Acknowledged scientists like Espen Tønnessen, Stephen Seiler, and Øyvind Sandbakk have been key contributors to this. During the last decade, this research group has been accountable for more than 100 publications through the Olympiatoppen system. Their strategy has been to establish valid and reliable protocols for testing and training monitoring; describe the physiological capacity, mechanical properties, and training content performed by worldleading athletes to understand the demands of selected Olympic sports; and test the training models derived by the best practitioners and reveal the underlying mechanisms. Several doctoral theses have followed this structure to merge science and best practice, for example, "Endurance Training Organization in Elite Endurance Athletes-From Description of Best Practice Towards Individualized Prescription" by Øystein Sylta. ${ }^{1}$ This $\mathrm{PhD}$ project has been described by Editor Emeritus Carl Foster as the most significant scientific work in endurance training in the last 20 years. Note that the best-practice description specified in that thesis was already implemented in the training framework of Norwegian pioneer endurance coaches in the 1970s. Similarly, the very best coaches in sprint training also seem to be decades ahead of sport science in how training principles and methods are applied. ${ }^{2}$ Possible explanations for such discrepancies may be that scientific studies mainly examine isolated variables under standardized conditions, while best practice is concerned about external validity and applying a more holistic approach. To further close this gap between science and practice, future investigations should observe and assess elite athletes throughout the training year to establish mechanistic connections between training content, changes in performance, and underlying mechanical and physiological determinants.

The sport science community should always strive to verify what the best practitioners claim to have known for a long time, thereby providing underlying mechanisms for success factors and methods where it seems like the success comes despite this element. In both cases, science will improve practice! Although it is difficult to recruit elite athletes and coaches to research projects, the probability increases if they become involved in the research question outlining process. Moreover, "recipe books" and training guides based on the practical experience and intuition of world-leading coaches are often publicly available. ${ }^{2}$ Integration of available research evidence and results-proven practice provides a valid point of departure for outlining state-of-the-art training recommendations and generating new hypotheses to be tested in future research.

\section{Thomas Haugen, IJSPP Associate Editor, Kristiania University College}

\section{References}

1. Sylta $\emptyset$. Endurance Training Organization in Elite Endurance Athletes -From Description of Best Practice Towards Individualized Prescription [PhD thesis]. Kristiansand, Norway: University of Agder; 2017.

2. Haugen T, Seiler S, Sandbakk $\varnothing$, Tønnessen E. The training and development of elite sprint performance: an integration of scientific and best practice literature. Sports Med Open. 2019;5:44. PubMed ID: 31754845 doi:10.1186/s40798-019-0221-0 